\title{
TENTATIVA DE UTILIZAÇÃO DE VARIÁVEIS MORFOMÉTRICAS DE PERFIS DE VERTENTES PARA O ZONEAMENTO PRELIMINAR DO MEIO FÍSICO: O CASO DA FOLHA DE LEME, SÃO PAULO
}

\author{
José Augusto de LOLLO \\ Nilson GANDOLFI
}

\begin{abstract}
RESUMO
Os levantamentos de condições geotécnicas preliminares de grandes áreas, das quais se dispõe de poucas informações de prospecção geotécnica de subsolo, podem apresentar certas dificuldades na delimitação de unidades de materiais rochosos e, principalmente, materiais inconsolidados. O presente trabalho apresenta uma tentativa de utilização de descrição de forma das vertentes como critério preliminar de distinção entre materiais presentes na área estudada.
\end{abstract}

\section{ABSTRACT}

Establishment of preliminary geotecnical conditions of large areas in the absence of significant underground geotechnical surveys, may present difficulties in the definition of groups of materials, mainly of unconsolidated ones. This paper decribes an attempt to use the description of slope forms as a preliminary criterion for distinction among types of materials.

\section{INTRODUÇÃO}

Por se considerar que os trabalhos de campo representam uma etapa da cartografia geotécnica de alto custo, muito se tem discutido sobre a possibilidade de abreviar esta etapa substituindo-a parcialmente por algum mecanismo ágil de reconhecimento dos materiais.

A presente discussão mostra especial interesse quando o trabalho é realizado em escala regional, considerando-se grandes áreas do terreno. Apresenta-se aqui uma tentativa de utilização de variáveis morfométricas de perfis de vertentes como método básico de zoneamento preliminar de uma área.

Como não se tinha notícia de estudo similar realizado em território nacional, no contexto geológico estudado (Depressão Periférica da Bacia do Paraná), o trabalho teve um caráter de teste, não se podendo dispensar os de campo.

O processo de cartografia geotécnica foi executado com base na proposta metodológica de ZUQUETTE (1987) para trabalhos em escala regional. Os de campo proporcionaram um total de 405 pontos que, associados à informações anteriores de prospecção geotécnica, estudos de fotointerpretação e consulta a mapas geológicos disponíveis para a área ou que a abrangessem, permitiram um bom zoneamento dos materiais rochosos e inconsolidados existentes na escala adotada (1:50.000). O levantamento de materiais inconsolidados é apresentado no Mapa de Materiais Inconsolidados (Fig. 1).

A área estudada corresponde à Folha de Leme (SF-23-Y-A-II-1, IBGE, 1971) e as informações prévias foram obtidas a partir dos mapas de KAEFER (1979), ALMEIDA (1981), DAEE (1981) e LANDIM (1982), e de estudos geotécnicos executados pelo DER-SP, CESP, ESTATEC Fundações, SONDOSOLO, e análises contratadas pelos Departamentos de Águas e Energia Elétrica das Prefeituras de Leme e Pirassununga e pela Divisão de Engenharia da Caninha 51 S.A.

Após a elaboração desta etapa do trabalho, pôde-se então passar à caracterização das variáveis morfométricas de vertentes, as quais tiveram de obedecer aos princípios de simplicidade e rapidez de obtenção.

\section{MATERIAL E MÉTODOS}

Com base nos princípios já citados, foram escolhidas 12 variáveis que pudessem ser obtidas a partir de mapas topográficos e cuja determinação fosse rápida e fácil. 


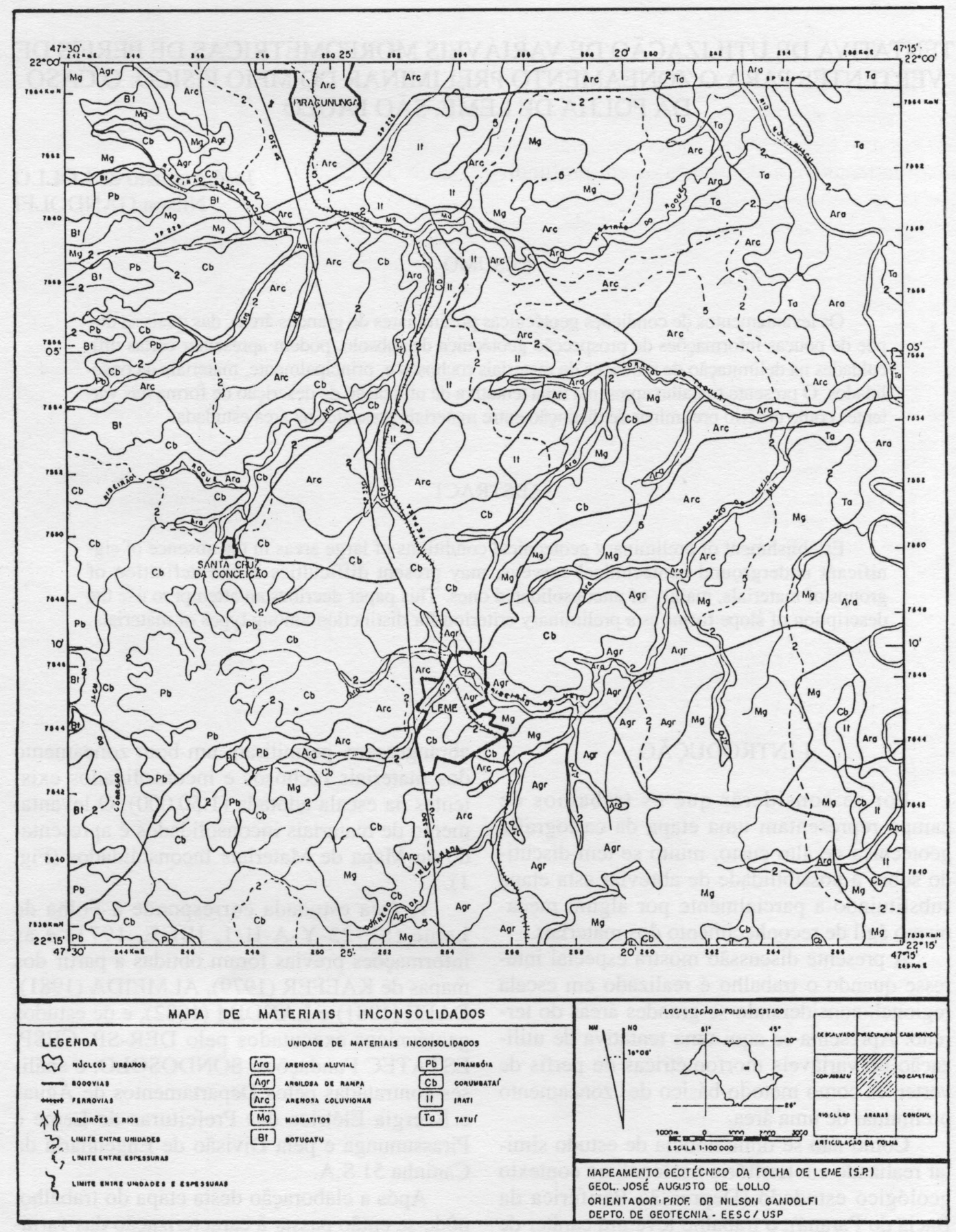

FIGURA 1 - Mapa de materiais inconsolidados 
As vertentes foram entendidas no sentido de DALRYMPLE et al. (apud CHRISTOFOLETTI, 1974) como um sistema tridimensional que se estende do interflúvio ao meio do canal fluvial e da superfície do terreno ao limite superior da rocha não intemperizada. A consideração, porém, da vertente como elemento tridimensional é bastante difícil em locais nos quais exista falta de informações das características de materiais inconsolidados em profundidade o que fez com que se optasse por utilizar o perfil longitudinal da vertente como objeto de estudo.

Esta unidade (perfil longitudinal) é obtida seccionando-se a vertente por um plano que lhe seja normal, que contenha sua linha de máximo declive, técnica proposta originalmente por SAVIGEAR (1956) e posteriormente desenvolvida por YOUNG (1964, 1971 e 1972).

Para que a escolha das vertentes não tivesse subjetividade, as vertentes foram selecionadas de forma aleatória, através da superposição, à área em estudo, de uma malha regular quadrada onde cada cela apresentava $1 \mathrm{~km}$ de lado, adotando-se para estudo a vertente que contivesse o ponto central da cela, segundo proposta de CHRISTOFOLETTI \& TAVARES (1977), proporcionando 672 vertentes.

Os dados foram levantados de forma a se obter dois conjuntos de coordenadas de pontos do perfil sendo representada em $\mathrm{x}$ a distância do ponto ao topo do perfil e em y a respectiva cota do ponto.

Para estes levantamentos as informações foram obtidas de mapas topográficos em escala 1:10.000 do Projeto Macrometrópole (IG, 1979) e, para garantir a rapidez dos cálculos e análises, foi desenvolvido um conjunto de aplicativos computacionais denominados VERTAN, BEST, VERTPAR, YOUNG e PROYOUNG (LOLLO, 1991).

Apesar de o número de variáveis morfométricas de perfis de vertentes descritas na literatura ser bastante grande, optou-se por aquela cujas definição pudesse ser rápida. Uma descrição pormenorizada das variáveis adotadas pode ser vista em SCHEIDEGGER (1961), TROECH (1965), CHRISTOFOLETTI ( 1974) e CHRISTOFOLETTI \& TAVARES (1976). De acordo com os princípios adotados, anteriormente citados, as variáveis escolhidas foram:

- Amplitude da Vertente (H): Diferença de cota entre os pontos de maior e menor cota do perfil (Fig. 2).

- Extensão da Vertente (L): Distância em planta entre os pontos de maior e menor cota do perfil (Fig. 2).

- Cumprimento Retilíneo (LR): Segmento de reta que une os pontos de maior e menor cota do perfil (Fig. 1).

- Cumprimento da Superfície (LS): Somatório dos comprimentos retilíneos de

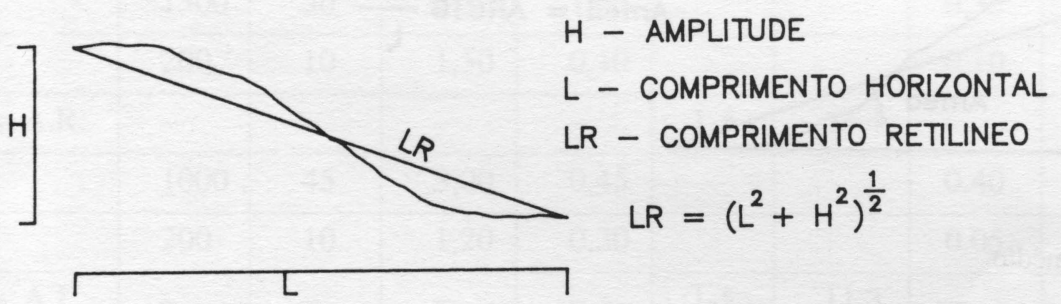

FIGURA 2 - Variáveis lineares

LS - COMPRIMENTO DA SUPERFICIE

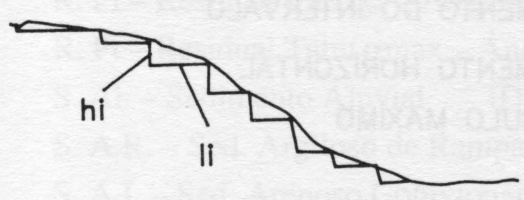

li - COMPRIMENTO DO INTERVALO

hi - AMPLITUDE DO INTERVALO

FIGURA 3 - Comprimento da superficíe 
todos os intervalos considerados no levantamento da vertente (Fig. 3).

- Índice de Retilinidade (IR): Razão entre o comprimento da superfície e o comprimento retilíneo da vertente.

- Ângulo Médio da Vertente (Amed): Ângulo entre os segmentos que definem o comprimento retilíneo e a extensão da vertente (Fig. 4).

- Ângulo Médio Ponderado (APmed): Média ponderada dos ângulos dos intervalos levantados na vertente (Fig. 4).

- Ângulo Máximo (Amax): Maior valor angular associado a um dos intervalos levantados da vertente, normalmente situado na porção retilínea do perfil (Fig. 5).

- Índice de Ruptura de Declive (ID): Número de pontos de inflexão presentes no perfil multiplicado por 100 e dividido por seu comprimento retilíneo.

- Coeficiente de Comprimento (CL): Razão entre os somatórios dos comprimentos dos intervalos da vertente posicionados em sua parte convexa (Lx) e em sua parte côncava (Lv).
- Coeficiente de Intensidade (CI): Razão entre os somatórios dos ângulos posicionados nas porções convexas do perfil (Ix) e nas porções côncavas (Iv).

- Índice de Intensidade (I): Razão entre os coeficientes de comprimento e intensidade da vertente.

\section{RESULTADOS}

O processo de levantamento de dados citado proporcionou 672 vertentes para análise. O cálculo das variáveis descritivas de forma da vertente foi efetuado a partir de um conjunto de utilitários computacionais gerenciados pelo programa VERTENTE (LOLLO, 1991).

Em seguida, as variáveis foram tabeladas, de duas formas: (1) unidades de material inconsolidado x intervalo de variação (Tab.1), (2) unidades geotécnicas (material inconsolidado / classe de espessura / substrato) $\mathrm{x}$ intervalos de variação (Tab. 2).

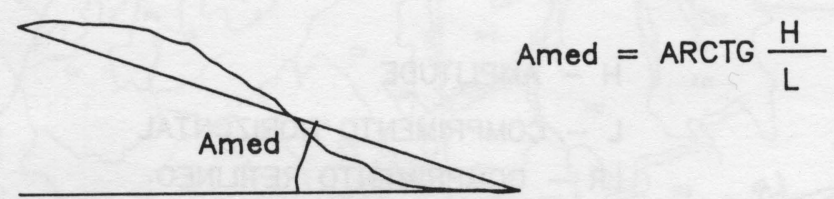

FIGURA 4 - Ângulo médio

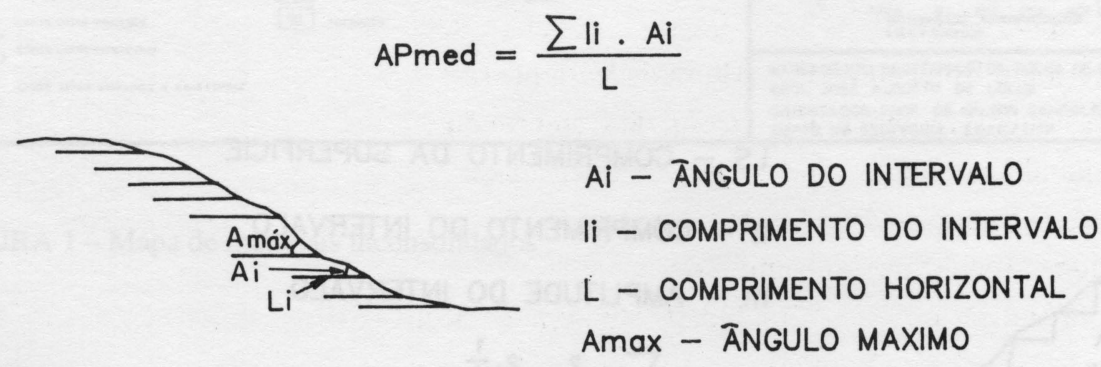

FIGURA 5 - Ângulo médio ponderado e ângulo máximo 
TABELA 1 - Intervalos dos Parâmetros - Materiais Inconsolidados

\begin{tabular}{|c|c|c|c|c|c|c|c|c|c|}
\hline Unidade & $\mathrm{L}$ & $\mathrm{H}$ & $\mathrm{CL}$ & $\mathrm{CI}$ & $\alpha^{\text {hn }}$ & $\alpha \max$ & ID & IF & $\mathrm{T}$ \\
\hline & 250 & 15 & 1,20 & 0,20 & & & 0,04 & 0,90 & \\
\hline \multirow{3}{*}{ R. Intr. } & - & - & - & - & $1-5$ & 26,6 & - & - & 1.0 \\
\hline & 1200 & 60 & 2,80 & 0,50 & & & 0,30 & 1,40 & \\
\hline & 250 & 30 & 0,80 & 0,10 & & & 0,28 & 0,75 & \\
\hline \multirow[t]{3}{*}{ R. TrJb } & - & - & - & - & $6-9$ & 26,6 & - & - & 1.0 \\
\hline & 600 & 70 & 1,40 & 0,30 & & & 0,40 & 1,20 & \\
\hline & 300 & 35 & 0,90 & 0,20 & & & 0,15 & 0,75 & \\
\hline \multirow[t]{3}{*}{ R. TrJp } & - & - & - & - & $4-10$ & 14,0 & - & - & 1.0 \\
\hline & 900 & 70 & 2,20 & 1,20 & & & 0,45 & 1,30 & \\
\hline & 400 & 20 & 1,20 & 0,20 & & & 0,05 & 0,90 & \\
\hline \multirow[t]{3}{*}{ R. Pc } & - & - & - & - & $2-4$ & 11,3 & - & - & 1.0 \\
\hline & 1200 & 85 & 2,40 & 0,60 & & & 0,25 & 1,40 & \\
\hline & 450 & 15 & 1,60 & 0,15 & & & 0,10 & 0,90 & \\
\hline \multirow[t]{3}{*}{ R. Pi } & - & - & - & - & $2-4$ & 8,1 & - & - & 1.0 \\
\hline & 1200 & 50 & 2,50 & 0,50 & & & 0,20 & 1,60 & \\
\hline & 300 & 15 & 1,10 & 0,25 & & & 0,10 & 0,80 & \\
\hline \multirow[t]{3}{*}{ R. Pt } & - & - & - & - & $2-3$ & 5,7 & - & - & 1.0 \\
\hline & 1100 & 45 & 2,50 & 0,70 & & & 0,35 & 1,90 & \\
\hline & 200 & 25 & 1,00 & 0,30 & & & 0,10 & 0,80 & \\
\hline \multirow[t]{3}{*}{ S. Al. } & - & - & - & - & $1-4$ & 5,7 & - & - & 1.0 \\
\hline & 1300 & 50 & 4,50 & 1,20 & & & 0,35 & 2,20 & \\
\hline & 200 & 10 & 1,50 & 0,10 & & & 0,10 & 1,00 & \\
\hline \multirow[t]{3}{*}{ s. A.R. } & - & - & - & - & $1-5$ & $18 ; 4$ & - & - & 1.0 \\
\hline & 1000 & 45 & 3,00 & 0,45 & & & 0,40 & 2,00 & \\
\hline & 200 & 10 & 1,20 & 0,30 & & & 0,05 & 0,90 & \\
\hline \multirow[t]{2}{*}{ s. A.I. } & - & - & - & - & $1-5$ & 11,3 & - & - & 1.0 \\
\hline & 1500 & 80 & 1,80 & 1,00 & & & 0,80 & 2,80 & \\
\hline
\end{tabular}

R. Intr. - Residual Magmatitos L - Compr. Horizontal (m)

R. TrJb - Residual Botucatu $\mathrm{H}$ - Altura da Vertente (m)

R. TrJp - Residual Pirambóia CL - Coef. de Comprimento

R. Pc - Residual Corumbataí CI - Coef. de Intensidade

R. Pi - Residual Irati $\alpha_{m}^{p}-$ Ângulo Med. Ponderado $\left({ }^{\circ}\right)$

R. Pt - Residual Tatuí $\alpha$ max - Ângulo Máximo $\left({ }^{\circ}\right)$

S. Al. - Sedimento Aluvial ID - Ind. de Ruptura de Declive

S. A.R. - Sed. Argiloso de Rampa IF - Índice de Forma

S. A.I. - Sed. Arenoso Coluvionar T - Tipo da Vertente (segundo CL e CI) 
TABELA 2 - Intervalos dos Parâmetros - Unidades Geoténicas

\begin{tabular}{|c|c|c|c|c|c|c|c|c|c|}
\hline Unidade & $\mathrm{L}$ & $\mathrm{H}$ & $\mathrm{CL}$ & $\mathrm{CI}$ & $\alpha_{\mathrm{m}}^{\mathrm{p}}$ & $\alpha \max$ & ID & IF & $T$ \\
\hline R. Intr. & 250 & 15 & 1,20 & 0,20 & & & 0,22 & 1,00 & \\
\hline & - & - & - & - & $2-5$ & 26,6 & - & - & 1.0 \\
\hline $\mathrm{e}<2 \mathrm{~m}$ & 450 & 35 & 1,75 & 0,35 & & & 0,30 & 1,40 & \\
\hline \multirow[t]{2}{*}{ R. Intr. } & 500 & 25 & 1,70 & 0,05 & & & 0,11 & 0,80 & \\
\hline & - & - & - & - & $1-4$ & 11,3 & - & - & 1.0 \\
\hline $\mathrm{e}: 2-5 \mathrm{~m}$ & 800 & 45 & 2,30 & 0,20 & & & 0,18 & 1,20 & \\
\hline \multirow[t]{2}{*}{ R. Intr. } & 900 & 35 & 2,00 & 0,30 & 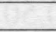 & & 0,04 & 0,90 & \\
\hline & - & - & - & - & $1-3$ & 11,3 & - & - & 1.0 \\
\hline$e>5 m$ & 1200 & 60 & 2,80 & 0,50 & ra & 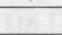 & 0,10 & 1,30 & \\
\hline \multirow[t]{2}{*}{ R. TrJb } & 350 & 30 & 0,80 & 0,10 & & & 0,28 & 0,75 & \\
\hline & - & - & - & - & $6-9$ & 26,6 & - & - & 1.0 \\
\hline$e<2 m$ & 600 & 70 & 1,40 & 0,30 & & & 0,40 & 1,20 & \\
\hline \multirow[t]{2}{*}{ R. TrJp } & 300 & 35 & 0,90 & 0,90 & & & 0,30 & 0,75 & \\
\hline & - & - & - & - & $5-10$ & 18,4 & - & - & 1.0 \\
\hline $\mathrm{e}<2 \mathrm{~m}$ & 500 & 50 & 1,40 & 1,20 & & & 0,45 & 1,00 & \\
\hline \multirow[t]{2}{*}{ R. TrJp } & 750 & 50 & 1,70 & 0,20 & & & 0,15 & 0,95 & \\
\hline & - & - & - & - & $4-7$ & 11,3 & - & - & 1.0 \\
\hline$e>2 m$ & 900 & 70 & 2,20 & 0,50 & & & 0,30 & 1,30 & 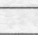 \\
\hline \multirow[t]{2}{*}{ R.Pc } & 400 & 20 & 1,20 & 0,35 & & & 0,15 & 0,90 & \\
\hline & - & - & - & - & $2-4$ & 14,0 & - & - & 1.0 \\
\hline $\mathrm{e}<2 \mathrm{~m}$ & 750 & 40 & 1,80 & 0,60 & & & 0,25 & 1,20 & 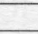 \\
\hline R. Pc & 900 & 55 & 2,00 & 0,20 & & 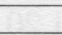 & 0,05 & 1,10 & \\
\hline & - & - & - & - & $2-4$ & 11,3 & - & - & 1.0 \\
\hline$e>2 m$ & 1200 & 85 & 2,40 & 0,40 & & & 0,20 & 1,40 & \\
\hline R.Pi & 450 & 15 & 2,20 & 0,30 & & & 0,10 & 1,30 & \\
\hline & - & - & - & - & $2-3$ & 5,7 & - & - & 1.0 \\
\hline $\mathrm{e}<2 \mathrm{~m}$ & 750 & 35 & 2,50 & 0,50 & & & 0,20 & 1,60 & 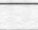 \\
\hline R. Pi & 900 & 30 & 1,60 & 0,15 & & & 0,10 & 0,90 & \\
\hline & - & - & - & - & $2-4$ & 8,1 & - & - & 1.0 \\
\hline$e>2 m$ & 1200 & 50 & 1,90 & 0,35 & & & 0,20 & 1,20 & \\
\hline R.Pt & 300 & 10 & 2,30 & 0,25 & & & 0,20 & 1,60 & \\
\hline & - & - & - & - & $2-3$ & 4,8 & - & - & 1.0 \\
\hline $\mathrm{e}<2 \mathrm{~m}$ & 500 & 25 & 2,50 & 0,35 & 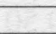 & 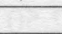 & 0,35 & 1,90 & \\
\hline R. Pt & 800 & 30 & 1,10 & 0,45 & & & 0,10 & 0,80 & \\
\hline & - & - & - & - & $2-3$ & 5,2 & - & - & 1.0 \\
\hline $\mathrm{e}>2 \mathrm{~m}$ & 1100 & 45 & 1,40 & 0,70 & & & 0,25 & 1,10 & \\
\hline S. Al./Int & 900 & 25 & 4,00 & 0,90 & & & 0,10 & 2,20 & \\
\hline & - & - & - & - & $1-3$ & 6,3 & - & - & 1.0 \\
\hline $\mathrm{e}<2 \mathrm{~m}$ & 1100 & 35 & 4,50 & 1,20 & & & 0,20 & 2,40 & \\
\hline S. Al./Pc & 200 & 30 & 1,00 & 0,30 & & & 0,25 & 0,80 & \\
\hline & - & - & - & - & $2-5$ & 8,1 & - & - & 1.0 \\
\hline $\mathrm{e}<2 \mathrm{~m}$ & 500 & 50 & 1,50 & 0,50 & & 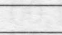 & 0,35 & 1,00 & \\
\hline S. Al./Pi & 400 & 20 & 2,00 & 0,30 & & & 1,10 & 0,90 & \\
\hline 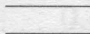 & - & - & - & - & $1-3$ & 5,7 & - & - & 1.0 \\
\hline $\mathrm{e}<2 \mathrm{~m}$ & 700 & 35 & 2,30 & 0,50 & & & 0,20 & 1,20 & \\
\hline S. Al./Pt & 800 & 30 & 1,00 & 0,60 & 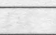 & & 0,15 & 0,90 & \\
\hline & - & - & - & - & $1-4$ & 7,1 & - & - & 1.0 \\
\hline $\mathrm{e}>2 \mathrm{~m}$ & 1300 & 50 & 1,40 & 0,90 & 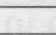 & 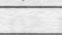 & 0,30 & 1,20 & \\
\hline SAR/Int & 200 & 10 & 2,20 & 0,20 & & & 0,20 & 1,00 & \\
\hline & - & - & - & - & $2-5$ & 8,1 & - & - & 1.0 \\
\hline $\mathrm{e}<2 \mathrm{~m}$ & 500 & 25 & 2,50 & 0,45 & & & 0,40 & 1,20 & \\
\hline SAR/Int & 700 & 20 & 1,50 & 0,10 & & & 0,10 & 1,50 & \\
\hline & - & - & - & - & $1-2$ & 8,1 & - & - & 1.0 \\
\hline$e>2 m$ & 1000 & 45 & 3,00 & 0,25 & & & 0,20 & 2,00 & \\
\hline SAI/Int & 300 & 10 & 1,20 & 0,15 & & & 0,15 & 0,90 & \\
\hline & - & - & - & - & $1-4$ & 6,3 & - & - & 1.0 \\
\hline$e<2 m$ & 600 & 30 & 1,50 & 0,40 & & & 0,30 & 1,20 & \\
\hline SAI/Int & 600 & 25 & 1,40 & 0,50 & & & 0,10 & 1,20 & \\
\hline & - & - & - & - & $1-3$ & 8,1 & - & - & 1.0 \\
\hline $\mathrm{e}: 2-5 \mathrm{~m}$ & 900 & 40 & 1,80 & 0,80 & & & 0,20 & 1,40 & \\
\hline SAI/Int & 1000 & 30 & 0,50 & 0,35 & & & 0,05 & 2,40 & \\
\hline & - & - & - & - & $1-3$ & 4,8 & - & - & 1.0 \\
\hline$e>5 m$ & 1500 & 55 & 0,90 & 0,60 & 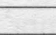 & 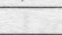 & 0,15 & 2,80 & \\
\hline $\mathrm{SAI} / \mathrm{Pc}$ & 200 & 25 & 1,40 & 0,70 & & & 0,20 & 1,80 & \\
\hline & - & - & - & - & $2-6$ & 11,3 & - & - & 1.0 \\
\hline$e<2 m$ & 500 & 45 & 1,80 & 1,00 & & & 0,40 & 2,20 & \\
\hline SAI/Pc & 600 & 40 & 1,30 & 0,50 & & $-\quad$ & 0,10 & 0,80 & \\
\hline & - & - & - & - & $2-5$ & 9,5 & - & - & 1.0 \\
\hline $\mathrm{e}: 2-5 \mathrm{~m}$ & 900 & 60 & 1,60 & 0,80 & & & 0,20 & 1,20 & \\
\hline SAI/Pc & 950 & 50 & 1,30 & 0,30 & & & 0,40 & 1,80 & 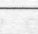 \\
\hline & - & - & - & - & $1-4$ & 9,5 & - & - & 1.0 \\
\hline$e>5 m$ & 1200 & 80 & 1,80 & 0,50 & & 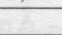 & 0,80 & 1,20 & \\
\hline$\overline{\mathrm{SAI} / \mathrm{Pi}}$ & 900 & 35 & 1,00 & 0,80 & & & 0,10 & 0,90 & \\
\hline & - & - & - & - & $2-3$ & 6,3 & - & - & 1.0 \\
\hline $\mathrm{e}>2 \mathrm{~m}$ & 1200 & 60 & 1,40 & 1,00 & & & 0,20 & 1,20 & \\
\hline
\end{tabular}

$\mathrm{SAI} / \mathrm{Pc}$ - inconsolidado / substrato geológico

Legenda: vide Tabela 1.

TABELA 2 - Intervalo dos Parâmetros - Unidades Geotécnicas 
Quanto às variáveis, pode-se afirmar o seguinte:

- Comprimento Retilíneo (LR) e Comprimento da Superfície (LS) não foram incluídas por apresentarem valores muito próximos aos da variável Extensão (L).

- Índice de Retilinidade (IR) não foi tabelada, pois apresentou valores muito próximos para todos os perfis analisados.

- Ângulo Médio (Amed) não foi incluída por apresentar valores muito próximos da variável Ângulo Médio Ponderado (APmed), a qual se encontra tabelada.

- Índice de Intensidade (I) não foi tabelada por se tratar de uma relação entre duas outras variáveis, apresentando, portanto, grande dispersão em termos de valores calculados.

\section{CONCLUSÕES}

A análise dos intervalos obtidos para as diversas unidades de materiais inconsolidados presentes proporcionou algumas observações interessantes acerca das variáveis usadas e seu uso como critério de distinção entre materiais inconsolidados ou classes de espessura:

- As variáveis CL (Coeficiente de Comprimento) e APmed (Ângulo Médio Ponderado) não se mostraram úteis para distinção entre unidades.

- Algumas das variáveis usadas refletiram aspectos esperados da evolução das vertentes: as variáveis L (Extensão) e H (Amplitude) apresentaram maiores valores para vertentes com maiores espessuras de materiais inconsolidados; a variável Amax (Ângulo Máximo) apresentou maiores valores para unidades com espessuras de materiais inconsolidados menores que $2 \mathrm{~m}$.

- Os intervalos de variação das variáveis CI (Coeficiente de Intensidade) e ID (Índice de Ruptura de Declive) proporcionaram uma boa distinção entre classes de espessura de materiais inconsolidados.

- As observações acima descritas permitem concluir que a aplicação destas variáveis morfométricas para tal finalidade apresenta restrições, visto que apenas duas delas parecem mostrar resultados interessantes; sugere-se, para trabalhos posteriores, a consideração tridimensional das formas do terreno.

\section{AGRADECIMENTOS}

Os autores gostariam de agradecer à FINEP pelos recursos fornecidos à pesquisa e à CAPES pela bolsa de estudos cedida.

\section{REFERÊNCIAS BIBLIOGRÁFICAS}

ALMEIDA, F.F.M. 1981. Mapa Geológico do Estado de São Paulo - escala 1:500.000. São Paulo, DMGA-IPT, Publicação 1184, Série Monografias, 6, 126p.

CHRISTOFOLETTI, A. 1974. Geomorfologia. São Paulo, Edgard Blucher, 150p.

CHRISTOFOLETTI, A. \& TAVARES, A.C. 1976. Análise de Perfis de Vertentes Esculpidas em Rochas do Grupo Nova Lima (Quadrilátero Ferrífero - Minas Gerais). Notícia Geomorfológica, Campinas, 16(31):41-56.

CHRISTOFOLETTI, A. \& TAVARES, A.C. 1977. Análise de Vertentes: caracterização e correção dos atributos do sistema. Notícia Geomorfológica, Campinas, 17(34):65-83.

DAEE 1981. Estudo da Qualidade de Águas Subterrâneas - Região Administrativa de Campinas. São Paulo, DAEE, 175p.

IBGE - Instituto Brasileiro de Geografia e Estatística. 1971. Mapa Topográfico: Folha de Leme (SF-23-Y-A-II-1). São Paulo, IBGE, Escala 1:50.000.

IG - Instituto Geológico. 1979. Projeto Macro-
Metrópole: 24 Mapas Topográficos. São Paulo, Secret. de Economia e Planejamento, Escala 1:10.000.

KAEFER, L.Q. 1979. Projeto Sapucaí: relatório final de geologia. CPRM Ministério de Minas e Energia. Série Geológica, Brasília, Boletim 2, 229p.

LANDIM, P.M.B. 1982. Mapa Geológico do Estado de São Paulo: Folha de Campinas (SF-23-Y-A). Escala 1:250.000. Rio Claro, DAEE/UNESP.

LOLLO, J.A. 1991. Mapeamento Geotécnico da Folha de Leme - SP: Utilização da Geomorfologia para a Caracterização Preliminar de Unidades Geotécnicas. Dissertação de Mestrado, Escola de Engenharia de São Carlos, 87p.

SAVIGEAR, R.A.G. 1956. Technique and Terminology in the Investigation of Slope Forms. Rio de Janeiro, Premier Rap. de la Comission pour le Etude des Versants, 66-75.

SCHEIDEGGER, A.E. 1961. Mathematical Models of Slope Development. Geological Society of America Bulletin, 72:37-50. 
TROECH, F.R. 1965. Landform Equations Fitted to Contour Maps. American Journal of Science, 263:616-627.

ZUQUETTE, L.V. 1987. Análise Crítica da Cartografia Geotécnica e Proposta Metodológica para as Condições Brasileiras. São Carlos: EESC/USP. 3.v. (Tese de Doutorado).

YOUNG, A. 1964. Slope Profile Analysis.
Zeitschrift fur Geomorphologie, Supplementband 5:17-27.

YOUNG, A. 1971. Slope Profile Analysis: the system of best units. In Slopes: form and processes, London, Institute of British Geographers Special Publication.

YOUNG, A. 1972. Slopes. London, Oliver \& Boyd, 251p.

Endereço dos autores:

José Augusto de LOLLO - Faculdade de Engenharia de Ilha Solteira - Av. Brasil Centro, 56 - Unesp - Caixa Postal 31 - $15378-000$ - Ilha Solteira - SP

Nilson Gandolfi - Escola de Engenharia de São Carlos - Universidade de São Paulo - Av. Dr. Carlos Botelho, 1465 - Caixa Postal 359 13560-970 - São Carlos - SP - Brasil. 